\title{
ISOMETRIC DEFORMATION OF THE SURFACE OF MINIMAL AREA OF A CYLINDER INCREASING ITS VOLUME
}

\author{
Lizandro Baldomero Reyna Zegarra y Heron Juan Morales Marchena \\ Departamento de Matemáticas, Universidad Nacional del Santa, Chimbote, Perú
}

\begin{abstract}
A method for increasing the volume of a cylinder with minimal area preserving the isometry is expounded. To do this, it was necessary to use numerical methods and a computer program. Such method let us set down a theorem which is analogy to that given for the case of a cube instead of a cylinder, being an immediate application of this theorem to the case of optimization in designing commercial bottles. To get an analytical proof of this new theorem is demanded. Keywords: surface of minimal area; optimization in designing commercial bottles; increasing the volume without stretching.
\end{abstract}

\section{INTRODUCCIÓN}

Recently, Igor Pak of the Massachusetts Institute of Technology - MIT has shown in a theorem [1] that there is a non-convex polyhedron whose surface is isometric to the surface of a cube of smaller volume. If we replace the cube by a right circular cylinder with minimal total area and the non-convex polyhedron by a solid of revolution of the non-convex type we are asking whether it is possible to find such a solid of revolution of the non-convex type with volume $V_{2}$ and area $A_{2}$ whose surface is isometric to that of the cylinder in such a way that if $V_{1}$ and $A_{1}$ are the volume and minimal area of a right circular cylinder we have $V_{1}<V_{2}$ and $A_{1}=A_{2}$. By isometric surfaces we mean those surfaces of equal constant Gaussian curvature [2],being in this particular case, zero. Here, the analogy to the meaning of isometric according to Pak is geodesic correspondence [2], which means to each geodesic on either surface there corresponds a geodesic on the other, being of course the geodesic distance between pairs of points on the surface of revolution of the nonconvex type generated by rotating a polygonal curve always equal to the geodesic distance between of the corresponding pairs of points on a cylinder.

The answer to this question is affirmative and in what follows the task is to show that it is so.
At the end it will be enounced as a theorem in analogy with the theorem in reference.

An outline of the plan that will let us prove the asseveration is shown in figure 1 .

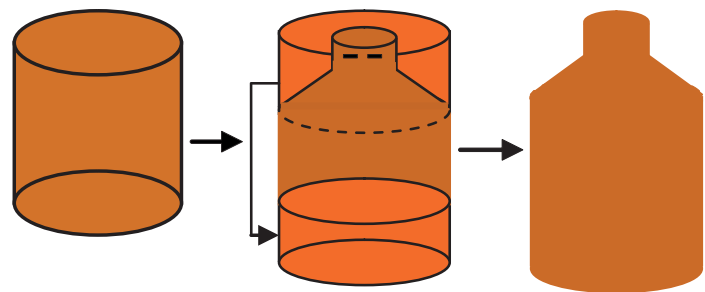

(a)

(b)

(c)

Figure 1 (a) Cylinder of minimal area (b) The external solid with internal limit, the surface of revolution, is removed and redistributed under a cylindrical shape in the below part of the cylinder without altering neither the superficial area nor the volume (c) Solid of revolution whose area is equal to that of the cylinder of minimal area.

Relation between the areas of a sphere, right circular cylinder of minimal area and a cube both of them enclosing the same volume.

The following proposition ${ }^{1}$ shows the inequality relation that is satisfied by the area of a sphere, the minimal area of a right circular cylinder and the area of a cube both of them enclosing the same volume $V$. 
Proposition: The areas of a sphere $A_{s p}$, of a right circular cylinder with minimal area $A_{c y}$ and of a cube $A_{c u}$ both of them enclosing the same volume $V$ are in such a way $A_{s p}<A_{c y}<A_{c u}$.

\section{Proof}

For the case of cube we have:

$$
\begin{gathered}
V=L^{3} \\
A_{c u}=6 L^{2}
\end{gathered}
$$

For the case of cylinder:

$V=\pi r^{2} h$ and since the cylinder has minimal area ${ }^{2}, h=2 r$ [4], hence $V=$ $\pi r^{2} h=\pi r^{2} .2 r=2 \pi r^{3}$, say,

$$
V=2 \pi r^{3}
$$

From (1) y (3) we have

$$
r=L \sqrt[3]{\frac{1}{2 \pi}}
$$

Furthermore the total area of the cylinder is given by $\mathrm{A}=2 \pi \mathrm{r}^{2}+2 \pi \mathrm{rh}$, and since $\mathrm{V}=\pi \mathrm{r}^{2} \mathrm{~h}$, we have

$$
A=2 \pi r^{2}+\frac{2 V}{r}
$$

By replacing (3) y (4) in (5), we have

$$
A=L^{2}\left[2 \pi \sqrt[3]{\frac{1}{4 \pi^{2}}}+2 \sqrt[3]{2 \pi}\right]
$$

From which

$$
A_{c y}=5.536 L^{2}
$$

For the case of the sphere:

$$
V=\frac{4}{3} \pi r^{3}
$$

From (1) and (8)

From which

$$
\mathrm{L}^{3}=\frac{4}{3} \pi \mathrm{r}^{3}
$$

$$
r=L \sqrt[3]{\frac{3}{4 \pi}}
$$

But the area of the sphere is $A=4 \pi r^{2}$. Then, according to (9) result

$$
A=4 \pi L^{2} \sqrt[3]{\frac{9}{16 \pi^{2}}}
$$

Say,

$$
A_{s p}=4.836 L^{2}
$$

Finally, from (2), (7) y (10) we have

$$
A_{\text {sp }}<A_{c y}<A_{c u}
$$

Hence the proposition is proved.

From (2), (7) y (10) we obtain the following relations:

$$
\frac{A_{c y}}{A_{s p}}=1.145 \quad, \quad \frac{A_{c u}}{A_{s p}}=1.241 \quad \frac{A_{c u}}{A_{c y}}=1.08
$$

Here we are interested in the first two relations. These ones show that we can build a cylinder having the same volume as it has the sphere but increased in an amount of area of $14.5 \%$. On the other hand, if we build a cube with the same volume of the sphere, the area is increased in an amount of $24.1 \%$. It is clear that the sphere is the geometrical object that encloses a maximum volume but having the smallest area.

Method for deforming isometrically the surface of minimal area of a cylinder increasing its volume.

\section{General method}

The method has two phases. The first one let us deform the cylinder of minimal area $A$ and volume $V_{2}$ to a solid of revolution of the nonconvex type with volume $V_{2}$ too, in such a way the value of its area $A_{2}$ be such that $A_{2}<A$.

The second one le tus build a cylinder of area $A_{1}$ and volume $V_{1}$ such that $A_{2}=A_{1}$ and show that $V_{1}<V_{2}$. This is equivalent to do an isometric deformation of the surface of a 
cylinder to a surface of a solid of revolution of the non-convex type in such a way that the volume that encloses the last one must be larger than the volume that encloses the first one.

In using of any software for industrial design it is possible to do what is illustrated in figure 1. However, we elaborated a computer program in Matlab 7.2 for this special case. The first phase of the general method involves the following sequence of steps [3], [5]:

1. In using differential calculus ${ }^{3}$, determine the radio $r$ and height $h$ of a right circular cylinder in such a way its superficial total area be minimal.

2. On a millimetered paper draw an $x y$ rectangular coordinate system, and on this one sketch the plane section that passes along the axis of the cylinder in 1 which by rotation generates the cylinder included its bases.

3. Taking into account indications in figure 1 , and after draw a set of points on the millimetered paper of point 2 in order to determine the shape of the generating curve that by rotation around the $x$ axis generates the surface of the solid of revolution of the non-convex type, in using numerical interpolation determine the curve that passes by the dotted line. See figure 2 .

4. By numerical integration determine the volume of the solid of revolution which by rotation generates the area under the curve obtained by numerical interpolation in 3 .

5. Make adjustments to the points drew in 3 in such a way that the value of the volume obtained by numerical integration be equal to the value of the volume of cylinder.

6. By numerical integration find the area of the surface of revolution that is generated by rotating the last curve obtained in the millimetered paper after carried out the adjustments to the points according to 5 .

7. Determine the difference of areas between the solid of revolution and the right cylinder

${ }^{3}$ Remember that the area as a function of the radius for the case of the cylinder is given by $A=2 \pi r^{2}+\frac{2 V}{r}$ of minimal area. This difference must be negative in the process of reducing the area of the first one (solid of revolution) to the second one (cylinder) under the condition that the value of the volume must remain fixed in the process. (In this process the curve that generates the surface of revolution will change of size horizontally and/or vertically keeping its shape).

8. Construct a new cylinder with a maximum volume enclosed by the area of the solid of revolution finally got in point 7 . This new cylinder is the cylinder that can be deformed isometrically to the solid of revolution of the non - convex type with the requirements we wish.

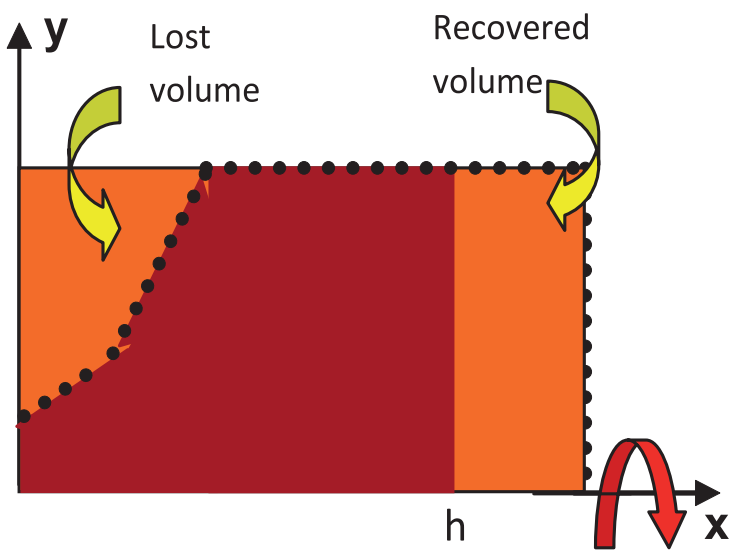

Figure 2

\section{Application of the method}

An example of application of the method here described is the following. Consider a cylinder of volume $V_{2}=537.5 \mathrm{~cm} 3$ and with the help of the computer program elaborated in Matlab (Figures 3, 4) we find the minimal area $A=365.96 \mathrm{~cm} 2$ that encloses such volume. Then, with the same computer program whose graphic interface is shown in figure 3 we proceed as it is illustrated in figure 2 and pointed out according to the steps 2 to 7 . The results are shown in table 2 for the first step, and table 3 for the second one. Figure 4 shows the solid of revolution obtained by rotating the polygonal curve of figure 3 whose coordinates are shown in table 1. 
Table 1

\begin{tabular}{|c|}
\hline Section 1 \\
\hline$(0,1.1),(1.5,1.1)$ \\
\hline Section 2 \\
\hline$(1.5,1.1),(2.5,3.2)$ \\
\hline Section 3 \\
\hline$(2.5,3.2),(3.5,4)$ \\
\hline Section 4 \\
\hline$(3.5,4),(5.5,3.67)$ \\
\hline Section 5 \\
\hline$(5.5,3.67),(7,4.45)$ \\
\hline Section 6 \\
\hline$(7,4.45),(8,4.5)$ \\
\hline Section 7 \\
\hline$(8,4.5),(8.3,4.4)$ \\
\hline Section 8 \\
\hline$(8.3,4.4),(9.71,4.4)$ \\
\hline Section 9 \\
\hline$(9.71,4.4),(11.01,4.5)$ \\
\hline Section 10 \\
\hline$(11.01,4.5),(12.01,3.988)$ \\
\hline
\end{tabular}

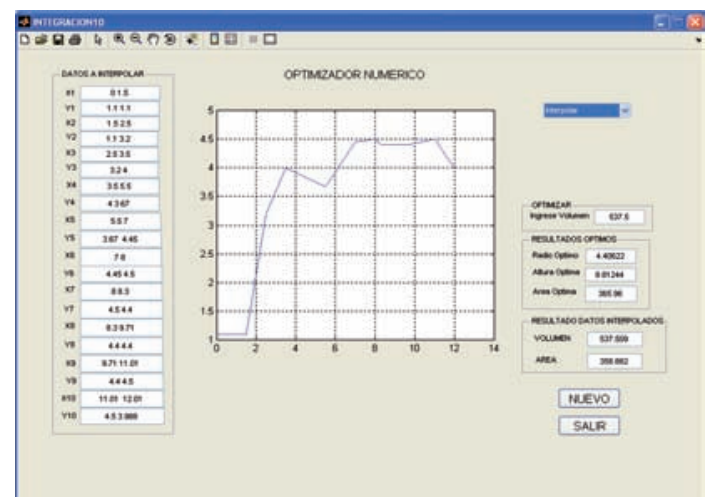

Figure 3
Table 2

\begin{tabular}{|c|c|c|c|}
\hline \multicolumn{2}{|c|}{$\begin{array}{l}\text { CYLINDER OF } \\
\text { MINIMAL AREA } \\
\text { INITIAL POINT } \\
\text { FROM WHICH } \\
\text { THE ISOMETRIC } \\
\text { DEFORMATION } \\
\text { STARTS }\end{array}$} & \multicolumn{2}{|c|}{$\begin{array}{l}\text { RESULT OF THE } \\
\text { NON-ISOMETRIC } \\
\text { DEFORMATION OF } \\
\text { THE CYLINDER TO } \\
\text { A SOLID OF } \\
\text { REVOLUTION OF } \\
\text { THE NON-CONVEX } \\
\text { TYPE }\end{array}$} \\
\hline $\begin{array}{l}\text { VOLUME } \\
\left(\mathrm{cm}^{3}\right)\end{array}$ & $\begin{array}{l}\text { AREA } \\
\left(\mathrm{cm}^{2}\right)\end{array}$ & $\begin{array}{l}\text { VOLUME } \\
\left(\mathrm{cm}^{3}\right)\end{array}$ & $\begin{array}{l}\text { AREA } \\
\left(\mathrm{cm}^{2}\right)\end{array}$ \\
\hline 537.5 & 365.96 & 537.509 & 358.882 \\
\hline
\end{tabular}

From $A=2 \pi r^{2}+\frac{2 V}{r}$, we solve for $\mathrm{V}$ and get

$$
\mathrm{V}=\frac{\mathrm{Ar}}{2}-\pi \mathrm{r}^{3}
$$

Due to the non-isometric deformation we got that the area of the solid of revolution of the non-convex type is equal to:

$$
A=358.882
$$

By replacing (2) in (1) we have

$$
V=179.441 r-\pi r^{3}
$$

Making use of differential calculus in order to determine the extremes of (3), it is found that the maximum happens at

$$
\mathrm{r}=4.3634 \mathrm{~cm}
$$

And it is unique.

By replacing (4) in (3) we obtain that the volume of the cylinder of area $A=358.882 \mathrm{~cm}^{2}$ is $V=521.9822 \mathrm{~cm}^{3}$.

Say, $V_{1}=521.9822 \mathrm{~cm}^{3}$, and since $V_{2}=537.5 \mathrm{~cm}^{3}$ we have $V_{1}<V_{2}$ as it was hoped.

The results are summarized in table 3 for the same area corresponding to the cylinder and to the solid of revolution of the non-convex type. 
Table 3

\begin{tabular}{|c|c|c|}
\hline \multicolumn{2}{|c|}{$\begin{array}{l}\text { FOR THE AREA OF THE } \\
\text { NEW CYLINDER AND } \\
\text { AREA OF THE SOLID OF } \\
\text { REVOLUTION OF THE } \\
\text { NON-CONVEX TYPE } \\
\text { BOTH OF THEM EQUALS } \\
\text { TO: } \\
358.882 \mathrm{~cm}^{2}\end{array}$} & $\begin{array}{l}\text { INCRE } \\
\text { MENT } \\
\left(\mathrm{cm}^{3}\right)\end{array}$ \\
\hline $\begin{array}{l}\text { VOLUME } \\
\text { OF THE } \\
\text { NEW } \\
\text { CILINDER } \\
\left(\mathrm{cm}^{3}\right)\end{array}$ & $\begin{array}{l}\text { VOLUME OF } \\
\text { THE SOLLID } \\
\text { OF } \\
\text { REVOLUTION } \\
\text { OF THE NON- } \\
\text { CONVEX } \\
\text { TYPE } \\
\left(\mathrm{cm}^{3}\right) \\
527509\end{array}$ & 15.527 \\
\hline 521.982 & & \\
\hline
\end{tabular}

From table 2 we see that the cylinder of minimal total area can be deformed to a solid of revolution of the non-convex type with less area than the initial cylinder. In this case, $\quad A=365.96 \mathrm{~cm} 2, \quad V_{2}=537.509 \mathrm{~cm} 3$ and $A_{2}=358.882 \mathrm{~cm} 2$. In this process the isometry is broken (it is not preserved), as we wished. In table 3 is shown the results for the volume and area of the new cylinder, $\mathrm{V}_{1}=521.982 \mathrm{~cm} 2$ y $A_{1}=358.882 \mathrm{~cm} 2$. Indeed the value $V_{2}=537.509 \mathrm{~cm} 3$ corresponding to the volume of the solid of revolution of the nonconvex type must be equal to $537.5 \mathrm{~cm} 3$. The slight difference obtained is a consequence of trying to get simultaneously $A_{2}<A$ keeping $V_{2}$ constant in the implementation of the first phase.

\section{RESULTS AND DISCUSSION}

The example of application of the method described shows that it is possible to deform isometrically the surface of a cylinder of minimal area to a surface of a solid of revolution of the non-convex type in such a way that the volume that enclose the last one is larger than the volume that encloses the first one.

Proof of proposition given above establishes that $A_{s p}<A_{c y}<A_{c u}$ where $A_{s p}, A_{c y}$ and $A_{c u}$ are the areas of sphere, cylinder and cube respectively, enclosing both of them the same volume and being these convex geometrical objects. The fact that it has been possible to show here that it is possible to build a solid of revolution of the non-convex type with area $A_{2}$ and volume $V_{2}$ such that if $A_{1}$ and $V_{1}$ are the area and volume of a right circular cylinder respectively, we have $A_{1}=A_{2}$ and $V_{1}<V_{2}$ is against the belief that only objects of the convex type always maximize the volume.

From Pak's theorem we can deduce that it is possible to increase the volume of a cube preserving the isometry until to get a volume that is $118 \%$ of the original volume, but in the case of the cylinder it remains as an open problem. This due that at first sight it is impossible to find a mathematical equation which let us get by analytical methods the necessary information as it is in the case of the cube.

Figure 5 and figure 6 show the isometric deformations for the case of cube and cylinder respectively and the corresponding analogy between both cases.

Figure 5
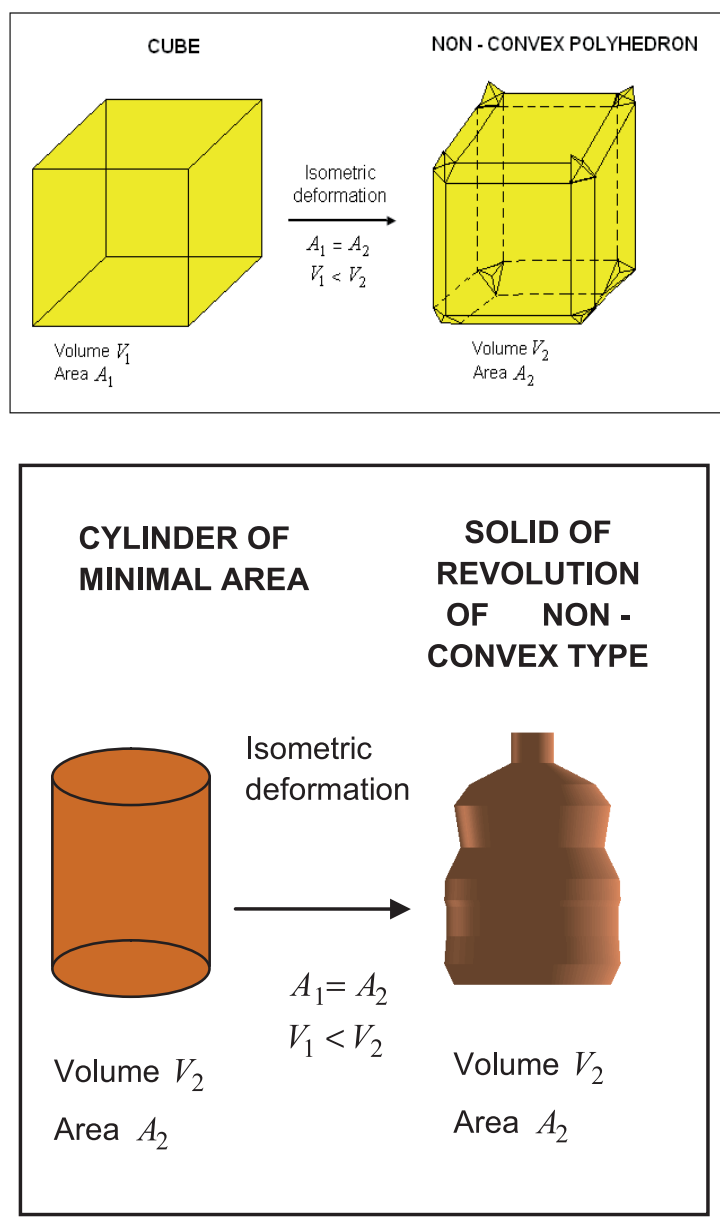

Figure 6 


\section{CONCLUSIONS}

The main conclusion arrived is that it is possible to find a solid of revolution of the non-convex type of volume $V_{2}$ and area $A_{2}$ in such a way that if $V_{1}$ and $A_{1}$ are the volume and area of a right circular cylinder of less volume, the relations $V_{1}<V_{2}$ and $A_{1}=A_{2}$ are satisfied. In analogy with Pak's theorem the following theorem is proposed:

THEOREM There exists a solid of revolution of the non-convex type whose surface is isometric to the surface of a right circular cylinder of minimal area and less volume than such solid of revolution.

The proof of the theorem in using a computer program follows the steps given above. However, an analytical proof would be nice.

As a first and immediate application of this theorem, corresponds to the industry of manufacturing of commercial bottles, since it will let us control the level of optimization in designing such bottles in order to minimize the use of plastic, glass, etc., avoiding contamination. In this case, the above theorem establishes a point of reference of extreme optimization but not absolutely because it could be an open problem yet.

\section{ACKNOWLEDGEMENTS}

It would not have been possible for us to write this article without the help of students of agroindustrial engineering of Universidad Nacional del Santa - Peru. We particularly want to thank Lopez Rivera, S. and Lavandera Alva, A. for their valuable contributions.

\section{REFERENCES}

[1] Pak, I. Inflating the Cube without Stretching arXiv:math. MO/067754 v1 28 Jul 2006.

[2] Pfahler, L An Introduction to Differential Geometrywith Use of the Tensor Calculus Princeton University Press 1947, pp.167,205,20.

[3] Reyna L.B, Moore T, Pajuelo A.L, et al. Criterio de Optimización por Compensación en la fabricación de envases con superficies laterales de revolución y bases planas Informe final de proyecto de investigación 2006 - UNS, Perú.

[4] Stein, S.K. Calculus and Analytic Geometry McGraw-hill, Inc., U.S.A 1982, pp.212-214.

[5] Eciperu, Enero - Junio 2009, Volumen 6, Numero 1, pp.63.

E - mail : reynazegarra@yahoo.es juanheron@hotmail.com 